persistent nitroglycerin treatment but terminated at least $4 \mathrm{~h}$ before ischaemia combined with intraperitoneal injection of ethanol $(0.5 \mathrm{~g} /$ $\mathrm{kg}$ ). Cardiac mitochondria was isolated after reperfusion. Three parameters were measured: (1) serum CK activity before ischaemia and after reperfusion; (2) after incubation with fluorescent probe JC-1, $\Delta \psi$ of isolated mitochondrial was evaluated by fluorometer and fluorescent microscope; (3) ALDH2 activity was determined spectrophotometrically by monitoring the reductive reaction of $\mathrm{NAD}^{+}$to $\mathrm{NADH}$ at $340 \mathrm{~nm}$.

Results (1) Ethanol treatment caused a $27 \%$ increase in ALDH2 activity relative to control $(2.74 \pm 0.37$ and $2.15 \pm 0.46, p=0.044)$; Treatment with persistent nitroglycerin decreased ALDH2 activity by $27 \%$ compared with ethanol treatment $(2.00 \pm 0.47$ and $2.74 \pm 0.37, p=0.024)$. There was no statistical difference in ALDH2 activity between GTN-ON and control group (2.00 \pm 0.47 and $2.15 \pm 0.46, p=0.642)$. (2) $\Delta \psi$ in cardiac myocytes in ethanol group and GTN-OFF group were 2.8 and 2.4 times higher than that in control respectively. $\Delta \psi$ in cardiac myocytes in GTN-ON group was reduced by $51 \%(119.33 \pm 10.21$ and $242.20 \pm 52.18, \mathrm{p}=0.006)$ and $42 \%(119.33 \pm 10.21$ and $204.00 \pm 86.17, p=0.045)$ than that in ethnol and GTN-OFF group respectively. (3) Cardiac damage was reduced by $39 \%(0.37 \pm 0.20$ and $0.61 \pm 0.12, p=0.029)$ and $41 \%(0.36 \pm 0.10$ and $0.61 \pm 0.12, p=0.031)$ in ethnol group and GTN-OFF group compared with that in control.

Conclusions Acute activation of ALDH2 reduced myocardial ischaemia/reperfusion injury possibly by preserving $\Delta \psi$.

\section{e0029 SURVIVAL OF TRANSPLANTED CELLS IN INFARCTED MYOCARDIUM AND EFFECT OF INDUCED NITRIC OXIDE SYNTHASE INHIBITOR}

doi:10.1136/hrt.2010.208967.29

Li Hongmin, Li Hongmin. Department of Cardiology Changzheng Hospital Affiliated To The Second Military Medical University Shanghai

Objective Cell transplantation was supposed to be a therapeutic strategy of myocardial infarction, but the problem was that most of the transplanted cells died at the early stage of transplantation. We hypothesised that over-activation of induced nitric oxide synthase (iNOS) may play an important role in the death of transplanted cells. In this experiment, we investigated the effect of iNOS selective inhibitor on the survival of transplanted bone marrow stem cells (BMSCs).

Methods BMSCs were isolated from the male SD rats. Female SD rats were subjected to myocardial infarction by ligation of left anterior descending artery. BMSCs at $1.2 \times 10^{6}$ were injected into the central zone of the infarct region at $1 \mathrm{~h}$ after ligation. BMSCs were monitored by labelled BrdU or Hoechst33342. A Sry gene sequence of Y chromatosome in male rats was analysed by real-time PCR for the quantification of the survived BMSCs in host myocardium.

Results The cultured BMSCs showed a homogenous pattern with CD44+/CD34-. Accumulation of BMSCs labelled with Hoechst33342 or BrdU was noted in infarct area of the host heart. Myocardial infarction induced a significant iNOS expression with its peak expression on day 3 and lasting for 14 days. The survival rates of engrafted BMSCs by real-time PCR were 7.88\%, 7.82\% and $8.73 \%$ at 1 week, 3 weeks and 6 weeks after transplantation respectively. iNOS selective inhibitor $1400 \mathrm{~W}$ contributed to $32 \%$ increase In the survival rate of the transplanted BMSCs. Migration of transplanted BMSCs to the margin area of infarct region was observed in Hoechst33342 or BrdU labelled BMSCs.

Conclusion 1 week after transplantation, the survival rate of BMSCs was as low as around $8 \%$ and there was no progressive decrease from 3 to 6 weeks post transplantation. iNOS selective inhibitor $1400 \mathrm{~W}$ may result in $32 \%$ increase in the survival rate of transplanted BMSCs.

\section{e0030 MESENCHYMAL STEM CELLS TRANSFECTED WITH HCN4 GENES BY LENTIV TO CREATE CARDIAC PACEMAKERS}

doi:10.1136/hrt.2010.208967.30

${ }^{1}$ Zhou Yafeng, ${ }^{2}$ Yang Xiangjun. ${ }^{1}$ Department of Cardiology, The First Affiliated Hospital of Soochow University; ${ }^{2}$ Department of Cardiology

Objective The study was to test proof-of-principle if genetically engineered mesenchymal stem cells (MSCs) transfected with HCN4 genes can be modified to be cardiac pacemaker cells.

Methods 1. MSCs of rabbit were isolated from the posterior iliac crest of rabbit and were used from passages 2 to 4 . 2. The self-inactivating HIV1-based lentiviral vector (LentiV) was used as transgene delivery, which was constructed with plasmid hHCN4/pcDNA3. 3. Total RNA was extracted from control MSCs and those transfected with hHCN4, and RT-PCR was performed. 4. Membrane proteins were extracted from control MSCs and those transfected with hHCN4. Western blot analysis was performed. 5 . Whole-cell patch clamp was used to study membrane currents. After the $\mathrm{I}_{\mathrm{f}}$ was recorded, cells were superfused with extracellular solution containing $4 \mathrm{mM}$ caesium chloride and the currents were measured accordingly. 6. MSCs transfected with either GFP alone or GFP-hHCN4 were cocultured with neonatal rabbit ventricular myocytes. The coculture beating rate of cardiac myocytes was measured after 3 days of coculture.

Results 1. In addition to expressing characteristic hHCN4 protein, mHCN4-transfected hMSCs also express an anticipated high level of hHCN4 gene by RT-PCR and Western blot analysis. 2. If was elicited using hyperpolarizing steps in $10-\mathrm{mV}$ increments from $-40 \mathrm{mV}$ to $-140 \mathrm{mV}$, and it was significantly inhibited by $4 \mathrm{mM}$ caesium chloride. 3. The coculture beating rate of cardiac myocytes was $87 \pm 11 \mathrm{bpm}$ when MSCs were transfected with control plasmid (expressing only GFP) and $149 \pm 14 \mathrm{bpm}$ when MSCs were expressing both GFP + hHCN4 $(\mathrm{p}<0.05)$.

Conclusion The MSC expressing hHCN4 is a demonstration of feasibility of preparing MSC-based biological pacemaker cells. The study provides a platform for further investigation of the possibility of modifying cardiac excitability with genetically engineered MSCs transfected with HCN4 gene by LentiV. MSCs transfected with hHCN4 genes by LentiV are potentially capable of actively pacing ventricular cardiac myocytes and can potentially be modified to be cardiac pacemaker cells.

\section{P0031 CHARACTER OF PACEMAKER CURRENT IN MESENCHYMAL STEM CELLS TRANSFECTED WITH HCN4 GENES}

doi:10.1136/hrt.2010.208967.31

Zhou Yafeng, Yang Xiangjun. Department of Cardiology, The First Affiliated Hospital of Soochow University, China

Objective To test if the pacemaker current can be elicited from mesenchymal stem cells (MSCs) transfected with HCN4 genes by LentiV.

Methods 1. MSCs of rabbit from the posterior iliac crest of rabbit were cultured at $37^{\circ} \mathrm{C}$ in a humidified atmosphere of $5 \% \mathrm{CO} 2.2$ The self-inactivating HIV-based lentiviral vector (LentiV) was used as transgene delivery, which was constructed with plasmid hHCN4/ pcDNA3. 3. Total RNA was extracted from control MSCs and those transfected with hHCN4, and RT-PCR was performed. 4. Whole-cell patch clamp was used to study membrane currents. If were elicited in the whole cell configuration by holding cells at $-40 \mathrm{mV}$ for $50 \mathrm{~ms}$ followed by $10-\mathrm{mV}$ steps $(2 \mathrm{~s})$ to $-130 \mathrm{mV}$ and returned to $-40 \mathrm{mV}$ (50 ms) after each step. After the $\mathrm{I}_{\mathrm{f}}$ was recorded, cells were superfused with extracellular solution containing $4 \mathrm{mM}$ caesium chloride and the currents were measured accordingly. 